\title{
QUASI-EINSTEIN CONTACT METRIC MANIFOLDS
}

\author{
AMALENDU GHOSH \\ Department of Mathematics, Chandernagore College, \\ Chandannagar, 712 136, W.B. India \\ e-mail: aghosh_70@yahoo.com
}

(Received 4 October 2013; revised 22 November 2013; accepted 14 January 2014; first published online 18 December 2014)

\begin{abstract}
We consider quasi-Einstein metrics in the framework of contact metric manifolds and prove some rigidity results. First, we show that any quasi-Einstein Sasakian metric is Einstein. Next, we prove that any complete $K$-contact manifold with quasi-Einstein metric is compact Einstein and Sasakian. To this end, we extend these results for $(\kappa, \mu)$-spaces.
\end{abstract}

2010 Mathematics Subject Classification. 53C25, 53C15, 53C21.

1. Introduction. A Riemannian manifold $(M, g)$ is said to be Einstein if the Ricci tensor $S$ of $M$ is a constant multiple of the metric tensor, i.e. $S=\lambda g$. In the framework of contact geometry, the Einstein condition and its several generalizations have been studied (e.g. see $[6,10-12,18])$. A careful study of Einstein $K$-contact and $\eta$-Einstein $K$ contact manifolds has been done by Boyer-Galicki [6]. In particular, they proved that $a$ compact Einstein $K$-contact manifold is Sasakian. This result is also true for $\eta$-Einstein (i.e. $S=\alpha g+\beta \eta \otimes \eta$, for some constants $\alpha, \beta$ and $\eta$ is the contact form) $K$-contact manifolds with $\alpha \geq-2$. One may consider this as an odd dimensional analogue of Goldberg conjecture, which says that a compact almost Kaehler Einstein manifold is Kaehler (see [3]). Recently, Sharma [18] pointed out that these results are still valid if one relax the hypothesis compactness to completeness. We also note that for a general contact metric manifold, the odd dimensional Goldberg conjecture is not true (see Apostolov-Drăghichi-Moroianu [1]).

A Ricci soliton is a natural generalization of an Einstein metric. It is defined to be a Riemannian manifold $(M, g)$ together with a vector field $V$ and a constant $\lambda$ that satisfies

$$
£_{V} g+2 S=2 \lambda g
$$

where $£_{V}$ denotes the Lie-derivative operator along the vector field $V$. Actually, it arises as a fixed point of the Hamilton's [13] Ricci flow: $\frac{\partial}{\partial t} g=-2 S$, viewed as a dynamical system on the space of Riemannian metrics modulo diffeomorphisms and scalings. The Ricci soliton is said to be shrinking, steady and expanding when $\lambda$ is positive, zero, and negative, respectively. If the vector field $V$ is Killing, the soliton becomes trivial (Einstein). Moreover, if $V=D f$, where $f$ is a smooth function on $M$ and $D$ is the gradient operator, then it is called a gradient Ricci soliton. Perelman [15] showed that all compact Ricci solitons can be realized as gradient Ricci solitons. For details about Ricci soliton, we refer to the survey paper [7] and the references therein. 
Several aspects of contact metrics as Ricci solitons have been studied by Sharma [18], Ghosh-Sharma-Cho [12], Cho-Sharma [10] and the author [11].

Recently, an interesting generalization of Einstein metrics which is closely related to the warped product spaces has been considered (see $[\mathbf{8}, \mathbf{9}, \mathbf{1 4}])$. This generalization arises from the $m$-Bakry-Emery Ricci tensor. Such tensor is defined as follows:

$$
S_{f}^{m}=S+\nabla^{2} f-\frac{1}{m} d f \otimes d f
$$

where $0<m \leq \infty, \nabla^{2} f$ denotes the Hessian form of the smooth function $f$ and $S$ denotes the Ricci tensor of $g$. This tensor arises from the warped product $(M \times N, \bar{g})$ of two Riemannian manifolds $\left(M^{n}, g\right)$ and $\left(N^{m}, h\right)$ with the Riemannian metric

$$
\bar{g}=g+e^{\frac{-2 f}{m}} h .
$$

Moreover, $\bar{g}$ is Einstein if and only if $(N, h)$ is Einstein, $S_{f}^{m}=\lambda g$, and a particular relationship holds between the scalar curvature of $(N, h)$ and the function $f$ (see [2]). We say that $(M, g, f, m)$ is a quasi-Einstein if there exists a constant $\lambda$ on $M$ such that

$$
S_{f}^{m}=S+\nabla^{2} f-\frac{1}{m} d f \otimes d f=\lambda g .
$$

When $f$ is constant, this gives the usual Einstein condition. For $m=1$, quasi-Einstein metrics are usually known as static metrics. These metrics have been studied in depth for their connections to scalar curvature, the positive mass theorem and general relativity. Note also that equation (1.1) recovers the gradient Ricci soliton condition when $m=$ $\infty$. In [9], Case et al. generalized some rigidity results of gradient Ricci solitons which were proved by Petersen-Wylie [16]. Some non-existence results for quasi-Einstein metric were also obtained in [8].

For a $K$-contact metric, Sharma [18] proved that if a complete $K$-contact metric is a gradient Ricci soliton, then it is a compact Einstein Sasakian manifold and the soliton is expanding. This result is also true for Sasakian manifolds as $K$-contact manifold contains the Sasakian manifold. In [12], Ghosh et al. extended this result for $(\kappa, \mu)$ spaces by proving that if the metric of a non-K-contact $(\kappa, \mu)$-space is a gradient Ricci soliton, then in dimension 3 it is locally flat, and in higher dimensions it is locally isometric to $E^{n+1} \times S^{n}(4)$. Further, this result has been generalized by Cho-Sharma [10] for homogeneous $H$-contact manifolds. From these results, we are inspired to study the quasi-Einstein condition for Sasakian and $K$-contact manifold. First, we prove

THEOREM 1. Let $M^{2 n+1}(\varphi, \xi, \eta, g)$ be a Sasakian manifold. If there exists a quasiEinstein structure $(g, f, m)$ associated with the contact metric $g$, then $M$ is Einstein and $f$ is constant.

Next, we consider $K$-contact metrics with quasi-Einstein condition for finite $m$ and present a generalization of Boyer-Galicki's result on $K$-contact Einstein manifold.

THEOREM 2. Let $M^{2 n+1}(\varphi, \xi, \eta, g)$ be a complete $K$-contact manifold. If there exists a quasi-Einstein structure $(g, f, m)$ associated with the contact metric $g$, then $f$ is constant and $M$ is a compact, Einstein, and Sasakian.

Finally, we extend these results for $(\kappa, \mu)$-spaces with quasi-Einstein condition and prove 
THEOREM 3. Let $M^{2 n+1}(\varphi, \xi, \eta, g)$ be a $(\kappa, \mu)$-space. Suppose there exists a quasiEinstein structure $(g, f, m)$ associated with the metric $g$. If $n=1$, then it is a space form including flat, while otherwise it is either locally isometric to $E^{n+1} \times S^{n}(4)$ or Einstein Sasakian.

2. Preliminaries. In this section, we shall recall the basic definitions and formulas of contact metrics and $K$-contact manifolds. An $(2 n+1)$ dimensional Riemannian manifold is said to be a contact manifold if it has a global 1-form $\eta$ such that $\eta \wedge(d \eta)^{n}$ is non-vanishing everywhere on $M$. We call this 1 -form a contact 1-form. For this 1form $\eta$ one can find a unit vector field $\xi$, called the Reeb vector field, such that $\eta(\xi)=1$ and $d \eta(\xi,)=$.0 . Further, there exists a Riemannian metric $g$ and a $(1-1)$ tensor field $\varphi$ satisfying

$$
d \eta(X, Y)=g(X, \varphi Y), \quad \eta(X)=g(X, \xi), \quad \varphi^{2} X=-X+\eta(X) \xi .
$$

These equations also imply

$$
\varphi \xi=0, \quad \eta \circ \varphi=0, \quad g(\varphi X, \varphi Y)=g(X, Y)-\eta(X) \eta(Y) .
$$

A Riemannian manifold together with the structure $(\varphi, \xi, \eta, g)$ is said to be a contact metric manifold. Following [3] we define two self-adjoint operators $h$ and $l$ by $h=$ $\frac{1}{2}\left(£_{\xi} \varphi\right)$ and $l=R(., \xi) \xi$, respectively. These operators also satisfy:

$$
h \xi=0=l \xi, h \varphi=-\varphi h, \operatorname{Tr} h=\operatorname{Tr} h \varphi=0 .
$$

A contact metric manifold is said to be $K$-contact if $\xi$ is Killing (i.e. $h=0$, or $\operatorname{Tr} l=2 n$ ). The following formulas are valid for a $K$-contact manifold:

$$
\begin{aligned}
\nabla_{X} \xi & =-\varphi X, \\
Q \xi & =2 n \xi \\
R(X, \xi) \xi & =X-\eta(X) \xi
\end{aligned}
$$

where $\nabla$ is the operator of covariant differentiation of $g, Q$ the Ricci operator associated with the $(0,2)$ Ricci tensor $S$ and $R$ the Riemann curvature tensor of $g$. A contact metric manifold is said to be Sasakian if the metric cone $C(M)\left(d r^{2}+r^{2} g, d\left(r^{2} \eta\right)\right)$ is Kaehler (see [6]). Equivalently, a contact metric manifold is said to be Sasakian if and only if the curvature tensor $R$ satisfies

$$
R(X, Y) \xi=\eta(Y) X-\eta(X) Y .
$$

We also remark that a Sasakian manifold is $K$-contact, and the converse is true only in dimension 3.

A contact metric manifold $M^{2 n+1}(\varphi, \xi, \eta, g)$ is said to be $(\kappa, \mu)$-space if the curvature tensor satisfies [4]

$$
R(X, Y) \xi=\kappa\{\eta(Y) X-\eta(X) Y\}+\mu\{\eta(Y) h X-\eta(X) h Y\},
$$

for some $(\kappa, \mu) \in \mathbb{R}^{2}$. This type of space arises through a $D$-homothetic deformation [19] to a contact metric manifold which satisfies $R(X, Y) \xi=0$. The class of $(\kappa, \mu)$-spaces covers Sasakian manifolds (for $\kappa=1$ ) and the trivial sphere bundle 
$E^{n+1} \times S^{n}(4)$ (for $\kappa=\mu=0$ ). Examples of non-Sasakian $(\kappa, \mu)$-spaces are the tangent sphere bundles of Riemannian manifolds of constant curvature $\neq 1$. On one hand the equation (2.5) determines the curvature completely for $\kappa<1$ and on the other it is also invariant under $D$-homothetic deformation (see Tanno [19]). For $\kappa<1$ the Ricci tensor has the following form

$$
\begin{aligned}
Q X= & {[2(n-1)-n \mu] X+[2(n-1)+\mu] h X } \\
& +[2(1-n)+n(2 \kappa+\mu)] \eta(X) \xi .
\end{aligned}
$$

The following formula also holds for a $(\kappa, \mu)$-space

$$
h^{2}=(\kappa-1) \varphi^{2},
$$

where $\kappa \leq 1$. We also note that the scalar curvature $r$ of such class is given by

$$
r=2 n(2(n-1)+\kappa-n \mu)
$$

and which is constant.

3. Proofs of the theorems. For the proofs of our results, we require the following.

LEMMA 1. For a quasi-Einstein metric the following formulae are valid

$$
\begin{aligned}
\text { (i) } R(X, Y) D f= & \left(\nabla_{Y} Q\right) X-\left(\nabla_{X} Q\right) Y+\lambda \frac{1}{m}[(Y f) X-(X f) Y] \\
& +\frac{1}{m}[(X f) Q Y-(Y f) Q X] . \\
\text { (ii) } S(Y, D f)= & \frac{1}{2}(Y r)+\frac{2 n \lambda}{m}(Y f)+\frac{1}{m}((Q Y) f)-\frac{r}{m}(Y f) .
\end{aligned}
$$

Proof. First, we note that equation (1.1) may be exhibited as

$$
\nabla_{Y} D f+Q Y-\frac{1}{m}(Y f) D f=\lambda Y .
$$

By straightforward computations, using the well-known expression of the curvature tensor:

$$
R(X, Y)=\nabla_{X} \nabla_{Y}-\nabla_{Y} \nabla_{X}-\nabla_{[X, Y]},
$$

and the repeated use of equation (3.3) gives equation (i). Next, contracting (3.1) over $X$ we get equation (ii). This completes the proof.

LEMMA 2. If $f$ is a smooth function on a contact metric manifold $M$ such that $d f=(\xi f) \eta$ (where d denotes the operator of exterior differentiation), then $f$ is constant on $M$.

Proof. Applying $d$ to the differential condition mentioned in the hypothesis and using the Poincare lemma : $d^{2}=0$ gives $d(\xi f) \wedge \eta+(\xi f) d \eta=0$. Taking wedge product of this equation with $\eta$ and noting that $\eta \wedge \eta=0$ and $d \eta \wedge \eta$ is non-vanishing everywhere on $M$, we conclude that $\xi f=0$. Consequently, $d f=0$ on $M$, and hence $f$ is constant on $M$, completing the proof. 
Proof of Theorem 1. Taking covariant differentiation of (2.2) and using (2.1) we get

$$
\left(\nabla_{X} Q\right) \xi=Q \varphi X-2 n \varphi X
$$

We know that in a Sasakian manifold the Ricci operator $Q$ and $\varphi$ commute each other, i.e. $Q \varphi=\varphi Q$ (see [3]). Thus, taking inner product of (3.1) and then making use of (2.2) and (3.4) gives

$$
\begin{aligned}
g(R(X, Y) D f, \xi)= & -2 g(Q \varphi X, Y)+4 n g(\varphi X, Y) \\
& +\frac{1}{m}(\lambda-2 n)[(Y f) \eta(X)-(X f) \eta(Y)]
\end{aligned}
$$

Substituting $Y$ by $\xi$ and recalling (2.3), equation (3.5) gives

$$
\left\{\frac{1}{m}(\lambda-2 n)-1\right\}[(Y f)-(\xi f) \eta(Y)]=0 .
$$

Since, $m$ and $\lambda$ are constant, we have either $\frac{1}{m}(\lambda-2 n)-1=0$, or $\frac{1}{m}(\lambda-2 n)-1 \neq 0$. We now discuss the two cases separately.

Case (i) When $\frac{1}{m}(\lambda-2 n)-1=0$. For a Sasakian manifold we know that $\xi$ is Killing and hence $£_{\xi} S=0$. Therefore, by (2.1) we easily get $\nabla_{\xi} Q=Q \varphi-\varphi Q$. For a Sasakian manifold $Q$ and $\varphi$ commute (see [3]) and hence $\nabla_{\xi} Q=0$. Now replacing $X$ by $\xi$ in (3.1), recalling the last relation, (2.2), (2.4) and (3.4) we find

$$
Q \varphi Y-2 n \varphi Y+\left\{\frac{1}{m}(\lambda-2 n)-1\right\}(Y f) \xi+\frac{\xi f}{m}\{Q Y+(m-\lambda) Y\}=0 .
$$

Taking inner product of this equation with $X$ and by virtue of $\frac{1}{m}(\lambda-2 n)=1$, one immediately finds

$$
g(Q \varphi Y-2 n \varphi Y, X)+\frac{1}{m}(\xi f) g(Q Y-2 n Y, X)=0 .
$$

Anti-symmetrizing (3.6) yields $(Q \varphi+\varphi Q) Y=4 n \varphi Y$, for all vector fields $X$. As $Q \varphi=$ $\varphi Q$ we have $Q \varphi Y=2 n \varphi Y$. Replacing $Y$ by $\varphi Y$ and recalling (2.2) it follows that $M$ is Einstein with constant scalar curvature $r=2 n(2 n+1)$. Since $M$ is Einstein and $\frac{1}{m}(\lambda-2 n)=1$, equation (3.2) yields $Y f=0$. This shows that $M$ is Einstein with $f$ constant.

Case (ii). When $(Y f)=(\xi f) \eta(Y)$. Applying Lemma 2, we can conclude that $f$ is constant on $M$. This finishes the proof.

Before proceeding to prove Theorem 2, we note that by a result of Qian [17], Case et al. [9] remark the following:

REMARK 3.1. A complete Riemannian manifold with quasi-Einstein metric is compact when $m$ is finite and $\lambda>0$.

Proof of Theorem 2. We distinguish the cases (i) $m=\infty$ and (ii) $0<m<\infty$. The first case together with (3.3) shows that the associated $K$-contact metric $g$ is a gradient Ricci soliton. Since $M$ is complete, the conclusion follows from the result of Sharma (see [18]). Next, we proceed to the case (ii). By virtue of (2.2) and (2.1), we note that 
$g\left(\left(\nabla_{X} Q\right) \xi, \xi\right)=0$ and $g\left(\left(\nabla_{\xi} Q\right) \xi, Y\right)=0$. Thus, taking inner product of (3.1) with $\xi$ and using (2.2), (2.3) we have

$$
\left\{\frac{1}{m}(\lambda-2 n)-1\right\}[(Y f)-(\xi f) \eta(Y)]=0 .
$$

Since $m, \lambda$ are all constant, from (3.7) we have either $\frac{1}{m}(\lambda-2 n)-1=0$, or $\frac{1}{m}(\lambda-$ $2 n)-1 \neq 0$. Making use of Lemma 2 , we can conclude that $f$ is constant, and hence $M$ is Einstein with scalar curvature $2 n(2 n+1)>0$. So, the compactness of $M$ follows from Myers' theorem. Applying the result of Boyer-Galicki [6], we can conclude that $M$ is Sasakian. So, it remains to consider the case $(\lambda-2 n)=m$. From (3.3) and (2.2) it follows that

$$
g\left(\nabla_{\xi} D f, \xi\right)=\lambda+\frac{(\xi f)^{2}}{m}-2 n .
$$

By definition, we have $g(\xi, D f)=\xi f$. Taking covariant differentiation of this along $\xi$ and noting that $\nabla_{\xi} \xi=0$ (follows from (2.1)), we obtain $g\left(\nabla_{\xi} D f, \xi\right)=\xi(\xi f)$. Hence,

$$
\xi(\xi f)=\lambda+\frac{(\xi f)^{2}}{m}-2 n .
$$

Since $\xi$ is Killing $\xi r=0$. Therefore, replacing $Y$ by $\xi$ in (3.2) we find that $(2 n \lambda-$ $2 n m+2 n-r)(\xi f)=0$. Using $\lambda=2 n+m$ the last equation transforms to $\{r-2 n(2 n+$ $1)\}(\xi f)=0$. Suppose $\{r-2 n(2 n+1)\}$ is non-vanishing in some open set $N$ of $M$. Then on $N$, we have $(\xi f)=0$. Making use of this in (3.8) shows that $\lambda=2 n$, and this leads to a contradiction as $m>0$. So, we must have $r=2 n(2 n+1)$, and clearly this is constant. Now, contract (3.3) to get

$$
\begin{aligned}
\triangle f & =r-\frac{1}{m}|D f|^{2}-(2 n+1) \lambda \\
& =2 n(2 n+1)-(2 n+1) \lambda-\frac{1}{m}|D f|^{2} \\
& =-(\lambda-2 n)(2 n+1)-\frac{1}{m}|D f|^{2},
\end{aligned}
$$

where we have used the convention $\Delta f=-\operatorname{div} D f$. Since $m$ is finite and $\lambda=m+2 n>0$, $M$ is compact by the above Remark. Applying the divergence theorem (see [20]) on (3.9) along with $m=\lambda-2 n$, we obtain

$$
\int_{M}|D f|^{2} d M=-(2 n+1) \int_{M}(\lambda-2 n)^{2} d M=-(2 n+1)(\lambda-2 n)^{2} \int_{M} d M .
$$

Now $\lambda$ being constant and the volume form $d M$ of a Riemannian manifold is always positive. Thus, the right hand side of the preceding equation is $\leq 0$. On the other hand, the integrand in the left hand side is non-negative. So we must have $D f=0$. This shows that $f$ is constant, which in turn implies that $M$ is Einstein. Applying BoyerGalicki's [6] result (stated earlier), we conclude that $M$ is Sasakian. This completes the proof. 


\section{Quasi-Einstein $(\kappa, \mu)$-spaces.} [3]:

Proof of Theorem 3. First, we note that for any contact metric manifold we have

$$
\nabla_{X} \xi=-\varphi X-\varphi h X
$$

and

$$
\nabla_{\xi} h=\varphi-\varphi h^{2}-\varphi l .
$$

From (2.6) we also have $Q \xi=2 n \kappa \xi$. Differentiating this along an arbitrary vector field $X$ and using (4.1) it follows that

$$
\left(\nabla_{X} Q\right) \xi=Q(\varphi+\varphi h) X-2 n \kappa(\varphi+\varphi h) X .
$$

Taking scalar product of (3.1) with $\xi$, using $Q \xi=2 n \kappa \xi$ we get

$$
\begin{aligned}
g(R(X, Y) D f, \xi)= & g\left(\left(\nabla_{Y} Q\right) X-\left(\nabla_{X} Q\right) Y, \xi\right) \\
& +\frac{\lambda-2 n \kappa}{m}[(Y f) \eta(X)-(X f) \eta(Y)] .
\end{aligned}
$$

Making use of (2.5), (4.3) in (4.4) and then replacing $X$ by $\varphi X$ and $Y$ by $\varphi Y$ yields

$$
(Q \varphi+\varphi Q) X-\varphi Q h X-h Q \varphi X-(4 n \kappa) \varphi X=0,
$$

for all vector field $X$ in $M$. Finally, applying (2.6) and (2.7) the last equation shows

$$
(\mu-2) \kappa=(n+1) \mu .
$$

Next taking $\xi$ instead of $Y$ in (4.4), recalling (2.5) and noting that $\left.\left(\nabla_{X} Q\right) \xi, \xi\right)=0$, $\left.\left(\nabla_{\xi} Q\right) X, \xi\right)=0$ (follows from (4.3)) we obtain

$$
h D f=\sigma[D f-(\xi f) \xi],
$$

where $\sigma=\frac{\lambda-2 n \kappa-m \kappa}{m \mu}$ and clearly this is constant. Differentiating (4.6) along an arbitrary vector field $X$ and taking into account (4.1), (3.3), (4.6), we find

$$
\begin{aligned}
& \left(\nabla_{X} h\right) D f-h Q X-\frac{\sigma(\xi f)}{m}(X f) \xi+\lambda h X \\
& \quad=\sigma[\lambda X-Q X-(X(\xi f)) \xi+(\xi f)(\varphi X+\varphi h X)] .
\end{aligned}
$$

Since $Q \xi=(2 n \kappa) \xi$, equation (3.3) provides

$$
\xi(\xi f)+2 n \kappa-\frac{(\xi f)^{2}}{m}=\lambda .
$$

On the other hand, from (2.5) it follows that $l=-\kappa \varphi^{2}+\mu h$. Using this and (2.7) in (4.2) we at once obtain $\nabla_{\xi} h=\mu h \varphi$. Replacing $X$ by $\xi$ in (4.7), using the last equation, $Q \xi=(2 n \kappa) \xi$ and (4.8) one immediately finds $\mu h D f=0$. This implies either (i) $\mu=0$, or (ii) $\mu \neq 0$.

Case (i). Using $\mu=0$ in (4.5) we see that $\kappa=0$. Thus $R(X, Y) \xi=0$, and hence $M$ is flat in dimension 3, and in higher dimensions $M$ is locally isometric to the trivial sphere bundle $E^{n+1} \times S^{n}(4)$ (see [3]). 
Case (ii). In this case, we have $h D f=0$. Using (2.7) one can easily deduce that

$$
0=h^{2} D f=(\kappa-1) \varphi^{2} D f .
$$

Since $\kappa \leq 1$, we have either $\kappa=1$, or $\kappa<1$. The former implies that $M$ is Sasakian and the latter shows that $D f=(\xi f) \xi$, and hence $f$ is constant, by Lemma 2. Consequently $M$ is Einstein, i.e. $Q X=(2 n \kappa) X$. For dimension 3, $M$ being Einstein it is of constant curvature 0 or 1 (see [5]). Next, since $M$ is Einstein it has constant scalar curvature $r=2 n \kappa(2 n+1)$. Comparing this with (2.8) it follows that $n \mu=2(n-1)-2 n \kappa$. By virtue of this and $Q X=(2 n \kappa) X$ we easily find from $(2.6)$ that $(2(n-1)+\mu) h=0$. If $(2(n-1)+\mu)$ is non-zero, then $h=0$, and, hence, $M$ is Einstein Sasakian. But if $2(n-1)+\mu=0$, then for $n=1, M$ is locally flat (as in this case $\mu=0=\kappa$, and, hence, $R(X, Y) \xi=0)$ and for $n>1$, using $\mu=2(1-n)$ in (4.5), we see that $\kappa=n-\frac{1}{n}>1$, a contradiction. This establishes the proof.

It is well known [9] that a compact Riemannian manifold with a quasi-Einstein metric with constant scalar curvature is Einstein. On the other hand, from (2.8) we see that the scalar curvature of a $(\kappa, \mu)$-space is constant. Thus, for a compact $(\kappa, \mu)$-space, and by virtue of the above theorem we have the following:

COROLlARYA 4.1. A compact non-Sasakian $(\kappa, \mu)$-space with a quasi-Einstein metric is flat and 3-dimensional with constant $f$.

Proof. Since $M$ is Einstein and non-Sasakian, from the previous theorem (see Case (ii)) it is obvious that $M$ is flat and 3-dimensional with constant $f$. This completes the proof.

ACKNOWLEDGEMENT. The author express his sincere thanks to the referee for many valuable suggestions.

\section{REFERENCES}

1. V. Apostolov, T. Drăghichi and A. Moroianu, The odd dimensional Goldberg conjecture, Math. Nachr. 279(9-10) (2006), 948-952.

2. A. L. Besse, Einstein manifolds (Springer, Berlin, 1987).

3. D. E. Blair, Riemannian geometry of contact and symplectic manifolds (Birkhauser, Boston, 2002).

4. D. E. Blair, T. Koufogiorgos and B. J. Papantoniou, Contact metric manifolds satisfying a nullity condition, Israel J. Math. 91(1-3) (1995), 189-214.

5. D. E. Blair and R. Sharma, Three dimensional locally symmetric contact metric manifolds, Boll. U.M.I. 4-A(7) (1990), 385-390.

6. C. P. Boyer and K. Galicki, Einstein manifolds and contact geometry, Proc. Am. Math. Soc. 129(8) (2001), 2419-2430.

7. H. D. Cao, Recent progress on Ricci soliton, Adv. Lect. Math. 11 (2009), 1-38. $227-284$

8. J. Case, On the non-existence of quasi-Einstein metrics, Pac. J. Math. 248(2) (2010),

9. J. Case, Y. Shu and G. Wei, Rigidity of quasi-Einstein metrics, Differ. Geom. Appl. 29(1) (2010), 93-100.

10. J. T. Cho and R. Sharma, Contact geometry and Ricci solitons, Int. J. Geom. Methods Math. Phys. 7(6) (2010), 951-960.

11. A. Ghosh, Ricci solitons and contact metric manifolds, Glasgow Math. J. 55(1) (2013), $123-130$. 
12. A. Ghosh, R. Sharma and J. T. Cho, Contact metric manifolds with $\eta$-parallel torsion tensor, Ann. Glob. Anal. Geom. 34(3) (2008), 287-299.

13. R. S. Hamilton, The Ricci flow: an introduction in: mathematics and general relativity (Santa Cruz, CA , 1986), Contemporary Mathematics, vol. 71 (American Mathematical Society, Providence RI, 1988), 237-262.

14. D.-S. Kim and Y. H. Kim, Compact Einstein warped product spaces with non-positive scalar curvature, Proc. Am. Math. Soc. 131(8) (2003), 2573-2576.

15. G. Perelman, The entropy formula for the Ricci flow and its geometric applications, Preprint, http:arXiv.org abs math.DG/02111159.

16. P. Petersen and W. Wylie, Rigidity of gradient Ricci solitons, Pacific J. Math. 241(2) (2009), 329-345.

17. Z. Qian, Estimates for weighted volumes and applications, Q. J. Math. Oxford Ser. 48(2) (1997), 235-242.

18. R. Sharma, Certain results on $K$-contact and $(k, \mu)$-contact manifolds, J. Geom. 89(1-2) (2008), 138-147.

19. S. Tanno, The topology of contact Riemannian manifolds, Illinois J. Math. 12(4) (1968), 700-717.

20. K. Yano, Integral formulas in Riemannian geometry (Marcel Dekker, New York, 1970). 